\title{
Aboriginal and Torres Strait Islander health: accuracy of patient self-report of screening for diabetes, high cholesterol and cervical cancer
}

\author{
Jessica Stewart, ${ }^{1,2}$ Rob Sanson-Fisher, ${ }^{1}$ Sandra Eades ${ }^{3}$
}

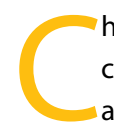
hronic diseases including diabetes, cardiovascular disease and cancer account for the majority of excess deaths and diseases among Aboriginal and Torres Strait Islander people, despite being largely preventable. ${ }^{1}$ Prevention activities such as regular screening are likely to produce significant health gains. ${ }^{2}$ To achieve these gains clinicians need appropriate, valid and reliable measures of a patient's screening history.

Patient self-report is often used by clinicians as a quick and inexpensive way to obtain information about a patient's screening status. Self-report is also used to determine the effectiveness of interventions intended to increase screening rates. The accuracy of selfreport is therefore critical. Research studies in non-Indigenous primary care settings, ${ }^{3-11}$ including Australia, ${ }^{12-15}$ have shown that relying on self-report can result in significant under-estimation of the proportion of people who require screening. Under-estimating the time period since a patient's last screening has been reported, especially among minority populations ${ }^{16,17}$ including indigenous native American women. ${ }^{18}$ Studies have also found that self-reported population survey data often under-estimates the prevalence of screening, particularly among marginalised population groups. ${ }^{9,10,19,20}$

Few published studies have investigated the validity of self-reported health issues among Aboriginal and Torres Strait Islander people in Australia. One study found self-reported

\begin{abstract}
Objective: To determine the accuracy and clinical utility of patient self-reported screening history.

Methods: Aboriginal and Torres Strait Islander patients ( $\geq 18$ years; $n=587$ ) were interviewed on their screening history according to recommended time intervals; these were matched to pathology data.

Results: The proportion of patients meeting screening guidelines were $32 \%(95 \% \mathrm{Cl} 26 \%-39 \%)$ for diabetes, $43 \%(95 \% \mathrm{Cl} 38 \%-47 \%)$ for cholesterol and $4.1 \%(95 \% \mathrm{Cl} 2.2 \%-7.3 \%)$ for cervical cancer. When patients reported having had the test, their accuracy (PPV) was low: $38 \%(95 \% \mathrm{Cl}$ $30 \%-46 \%)$ for diabetes, $47 \%(95 \% \mathrm{Cl} 42 \%-52 \%)$ for cholesterol, $6.5 \%(95 \% \mathrm{Cl} 3.0 \%-12 \%)$ for cervical cancer. However, for the minority of patients who had been screened, positive recall (sensitivity) was high: $94 \%(95 \% \mathrm{Cl} 85 \%-98 \%)$ for diabetes, $83 \%(95 \% \mathrm{Cl} 77 \%-88 \%)$ for cholesterol, $90 \%(95 \% \mathrm{Cl} 55 \%-98 \%)$ for cervical cancer. The accuracy of patient recall was good for those who reported not having been screened (NPV): $90 \%(95 \% \mathrm{Cl} 77 \%-97 \%)$ for diabetes, $70 \%(95 \% \mathrm{Cl} 61 \%-78 \%)$ for cholesterol, $99 \%(95 \% \mathrm{Cl} 95 \%-100 \%)$ for cervical cancer.

Conclusions: The results indicate that reliance on self-report for patients' screening history is inappropriate due to missed opportunities for health gain. However, patients who report not being tested are sufficiently accurate to ensure that ordering the test in this group will involve only a small percentage of unnecessary assays.

Implications: GPs often rely on self-report as a fast and inexpensive way to determine whether a patient requires screening. New strategies are needed to identify patients at risk.

Key words: Aboriginal and Torres Strait Islander people, accuracy self-report, chronic disease

information under-estimated the smoking status of pregnant Indigenous women, ${ }^{21}$ and another found a modest correlation between self-report and measured physical activity among Aboriginal children. ${ }^{22}$

This study aimed to examine the accuracy of patient self-reported screening status for diabetes, high cholesterol and cervical cancer among Aboriginal and Torres Strait Islander

patients when compared with pathology records. The study was undertaken in an Aboriginal Community Controlled Health Service (ACCHS). ACCHSs are culturally competent primary health care services and represent self-determination in the provision of health care. They are ideal settings for delivering prevention activities to Aboriginal and Torres Strait Islander people.
\end{abstract}

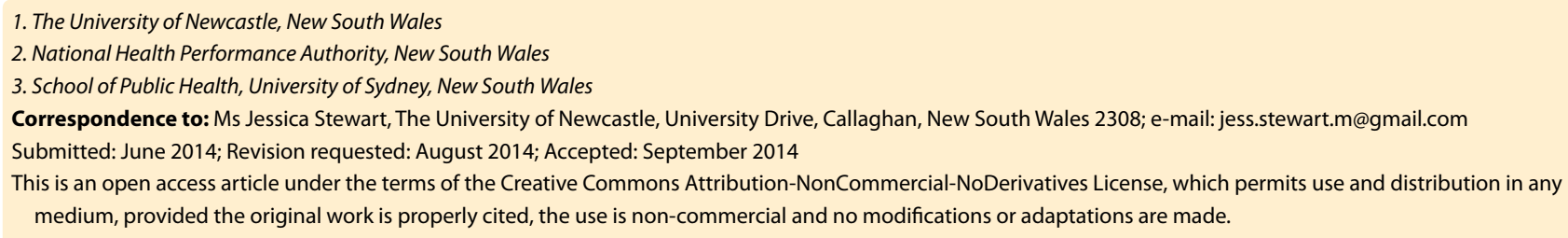




\section{Methods}

\section{Study design and setting}

This study was undertaken in three suburban ACCHS clinics within a large metropolitan region of Australia between November 2007 and July 2009. In May 2009, 13,116 patients (6,043 men and 7,078 women) were identified as current patients using electronic patient files (Communicare). The three clinics combined provided 47,468 episodes of care during the data collection period.

\section{Participants}

Study participants were recruited by research project officers and Aboriginal Health Workers at each clinic. All patients who identified as Aboriginal, Torres Strait Islander, or both, aged 18 years and over, who presented for a consultation with a health professional were eligible for inclusion in the study. Patients were excluded if they were non-Indigenous, were too ill (as determined by clinic staff), or if they had not been a client of the service for the duration of time stipulated by the relevant screening guideline (two years). On recruitment days, all patients on the appointment list were invited to participate. If workload prevented project officers approaching all patients, patients were identified by systematically sampling every second or third patient from the list, to reduce selection bias. The gender and age of nonconsenting patients was recorded.

\section{Collection of self-report data}

Informed consent was obtained from all participants for self-report information via a questionnaire and access to medical records. Data on patients' screening history were collected as part of a larger survey that also asked patients about self-reported behavioural health risk status and health concerns (described elsewhere). ${ }^{23}$ The survey asked patients about their risk status for behavioural risk factors and screening history for blood pressure, cholesterol, diabetes and cervical cancer. These items were chosen as they contribute significantly to the burden of disease among Aboriginal and Torres Strait Islander people, ${ }^{24}$ and there is evidence that regular screening can improve health outcomes. ${ }^{2}$

Included survey items were based on the most recent Australian Bureau of Statistics National Aboriginal and Torres Strait Islander Health Survey collected in 2004-05. ${ }^{25}$ The definitions of optimal screening schedules for patients were defined using current evidencebased guidelines for clinical preventive services in general practice. ${ }^{26-28}$

\section{Diabetes (type 2)}

Patients were asked, "When was the last time you had a blood test for diabetes?" Response categories were: less than one year ago; less than 3 years ago; more than 3 years ago; never; not sure. Patients were considered 'at-risk' if they were 35 years or over and had not had fasting blood sugar screening within the previous 12 months. Patients who self-reported having type 2 diabetes were excluded from the analysis of patients 'at-risk' of type 2 diabetes.

\section{Cholesterol}

Patients were asked, "When was the last time you had a blood test for cholesterol (blood fats)?" Response categories were: less than one year ago; $1-2$ years ago; in the past 5 years; more than 5 years ago; never; don't know. Patients were considered at risk if they were 18 years or older had not been screened within the previous two years as part of an overall cardiovascular risk status assessment.

\section{Cervical cancer}

Female patients were asked, "When was the last time you had a Pap smear?" A Pap smear was described as a simple test to check the health of women's cervix (the top of the womb) and the best way to find early warning signs that cancer might develop in the future. Response categories included: less than 2 years ago; more than 2 years ago; never. Women were considered at risk if they were between 18 and 69 years and had not had a Pap smear in the previous 2 years, and had not had a hysterectomy.

\section{Collection of pathology records}

Reports of pathology tests conducted during the study period were extracted from Communicare, the patient information management system used in each of the clinics. Communicare stores information on the patient's file on every pathology request sent to pathology laboratories. These reports include the date and type of test requested. Reports were extracted for all tests recorded within the study period for diabetes (type 2 ), cholesterol and cervical cancer, for all registered clients.

\section{Data analysis}

Patients' self-reported information on the time period since last screening were matched to pathology records using patient name, date of birth and patient identification number. Patients' responses of having been screened within the recommended time interval were compared with pathology test records for the same period. Responses were dichotomised into 'yes' if a pathology record was present, and 'no' if there was no pathology record present, of the test having been conducted within the recommended time interval. Sensitivity, specificity, and the positive and negative predictive values of selfreport as a measure of screening status were calculated with $95 \%$ confidence intervals by comparison with pathology records, which were considered the gold-standard.

\section{Ethics and community consultation}

The study was approved by the Human Research Ethics Committees of the Universities of Sydney and Newcastle, by the Western Australian Aboriginal Health Information and Ethics Committee and by the Boards of Management of the ACCHS in which the study was based.

\section{Results}

\section{Sample}

Of the 758 people assessed for eligibility and invited to join the study, 587 (77\%) consented, including 385 (66\%) women and 202 (34\%) men. There were no significant differences in age $\left(\chi^{2}=8.07, \mathrm{df}=4, p=0.089\right)$ between consenters and non-consenters. However the non-consenting group contained a greater proportion of males (45\%) than the consenting group $\left(\chi^{2}=6.42, \mathrm{df}=1, p=0.011\right)$.

\section{Diabetes (type 2)}

As shown in Table 1, 203 patients were eligible for inclusion to assess patient recall of screening for diabetes. Of these, only 65 (32\%; 95\%Cl 26\%-39\%) had a pathology test demonstrating that screening occurred according to the recommended guidelines.

The high sensitivity indicated that $94 \%$ $(95 \% \mathrm{Cl} 85 \%-98 \%)$ of patients who had a diabetes test recorded were able to accurately recall the test. Of patients reporting that they had not been screened for diabetes within the past 12 months, $90 \%$ (95\% Cl 77\%-97\%) had no pathology test recorded (NPV). However the very low specificity of $28 \%$ $(95 \% \mathrm{Cl} 20 \%-36 \%)$ indicates that $72 \%$ of 
patients who had no pathology test reported that they had been screened within the previous 12 months. The positive predictive value indicates that only $38 \%(95 \% \mathrm{Cl}$ $30 \%-46 \%$ ) of patients who reported having been screened were correct (Table 1).

\section{Cholesterol}

There were 460 patients eligible for inclusion to assess patient recall of screening for high cholesterol (Table 2). Of these, only 197 (43\%; 95\%Cl 38-47\%) had a pathology test confirming screening had occurred. Similar to diabetes screening, a patient's report that a test had been performed was unreliable with a PPV of $47 \%(95 \% \mathrm{Cl} 42 \%-52 \%)$, while a selfreport that the test had not been carried out was reasonably accurate with a NPV of $70 \%$ (95\% Cl 61\%-78\%)

The high sensitivity indicates that most patients $(83 \%$; $95 \% \mathrm{Cl} 77 \%-88 \%)$ who had a cholesterol test recorded were able to accurately recall the test. The specificity indicated that about one third $(30 \%(95 \% \mathrm{Cl}$ $25-36 \%)$ of patients with no test recorded were able to recall they had not been screened within the previous two years.

\section{Cervical cancer}

There were 246 female patients eligible for inclusion to assess patient recall of screening for cervical cancer. Of these, only 10 (4.1\%; $95 \% \mathrm{Cl} 2.2 \%-7.3 \%$ ) had a pathology test confirming screening had occurred. The high sensitivity indicates that $90 \%(95 \% \mathrm{Cl}$ $55 \%-98 \%$ ) of patients who had a Pap test recorded were able to accurately recall the test; however, the number of individuals with a Pap test recorded was very low (10), so the confidence interval for this estimate was very wide. The negative predictive values were very high, with $99 \%$ (95\%Cl 95\%-100\%) of patients stating that they had not had a Pap test, with no pathology record for this test. The specificity result of $45 \%$ ( $95 \% \mathrm{Cl} 38 \%$ $52 \%$ ) indicated that $55 \%$ of patients who had no pathology test reported that they had been screened within the previous two years. The positive predictive value indicated that only $6.5 \%(95 \% \mathrm{Cl} 3.0 \%-12 \%)$ of patients who reported having been screened were correct (Table 3).

\section{Discussion}

This study provides the first data on the accuracy of self-reported screening when verified against a pathology test record for

Table 1: Accuracy of patient self-report for diabetes screening relative to pathology records for Aboriginal and Torres Strait Islander people (>34 years) attending an Aboriginal Community Controlled Health Service.

Patient recal Pathology record indicating test within recommended guidelines*

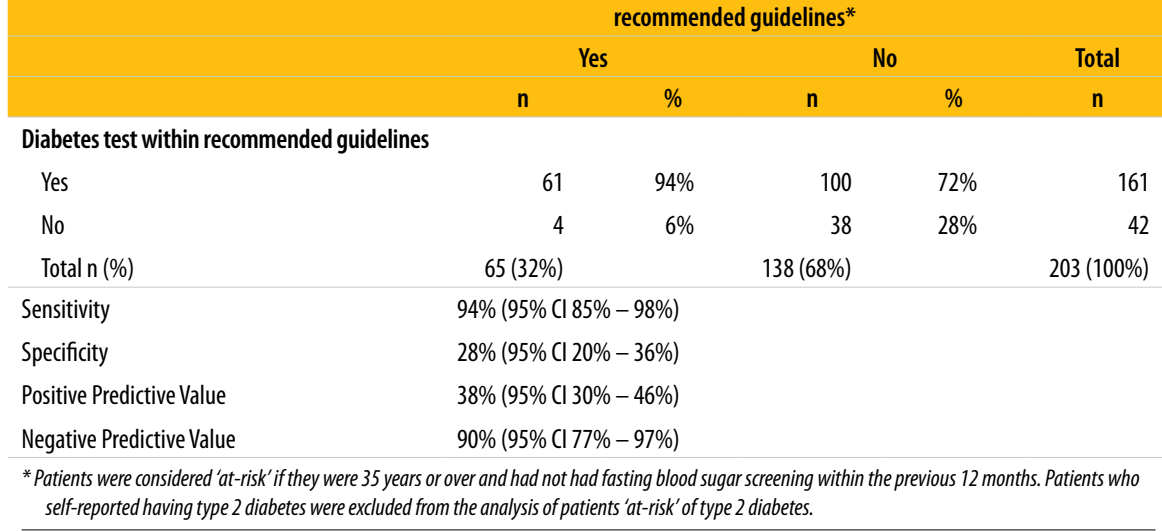

\begin{tabular}{|c|c|c|c|c|c|}
\hline \multirow[t]{3}{*}{ Patient recall } & \multicolumn{4}{|c|}{$\begin{array}{l}\text { Pathology record indicating test within } \\
\text { recommended guidelines* }\end{array}$} & \multirow{3}{*}{$\begin{array}{c}\text { Total } \\
\mathrm{n}\end{array}$} \\
\hline & \multicolumn{2}{|c|}{ Yes } & \multicolumn{2}{|c|}{ No } & \\
\hline & $\mathrm{n}$ & $\%$ & $\mathrm{n}$ & $\%$ & \\
\hline \multicolumn{6}{|c|}{ Cholesterol test within recommended guidelines } \\
\hline Yes & 163 & $83 \%$ & 184 & $70 \%$ & 347 \\
\hline No & 34 & $17 \%$ & 79 & $30 \%$ & 113 \\
\hline Total n (\%) & $197(43 \%)$ & & $263(57 \%)$ & & $460(100 \%)$ \\
\hline Sensitivity & \multicolumn{5}{|c|}{$83 \%(95 \%$ Cl $77 \%-87 \%)$} \\
\hline Specificity & \multicolumn{5}{|c|}{$30 \%(95 \%$ CI $25 \%-36 \%)$} \\
\hline Positive Predictive Value & \multicolumn{5}{|c|}{$47 \%(95 \%$ Cl $42 \%-52 \%)$} \\
\hline Negative Predictive Value & \multicolumn{5}{|c|}{$70 \%(95 \%$ Cl $61 \%-78 \%)$} \\
\hline
\end{tabular}

\begin{tabular}{|c|c|c|c|c|c|}
\hline \multirow[t]{3}{*}{ Patient recall } & \multicolumn{4}{|c|}{$\begin{array}{l}\text { Pathology record indicating test within } \\
\text { recommended guidelines* }\end{array}$} & \multirow{3}{*}{$\begin{array}{c}\text { Total } \\
\mathrm{n}\end{array}$} \\
\hline & \multicolumn{2}{|c|}{ Yes } & \multicolumn{2}{|c|}{ No } & \\
\hline & $\mathrm{n}$ & $\%$ & $\mathrm{n}$ & $\%$ & \\
\hline \multicolumn{6}{|c|}{ Pap test within recommended guidelines } \\
\hline Yes & 9 & $90 \%$ & 130 & $55 \%$ & 139 \\
\hline No & 1 & $10 \%$ & 106 & $45 \%$ & 107 \\
\hline Total n (\%) & $10(4.1 \%)$ & & $236(96 \%)$ & & $246(100 \%)$ \\
\hline Sensitivity & \multicolumn{5}{|c|}{$90 \%(95 \% \mathrm{Cl} 55 \%-98 \%)$} \\
\hline Specificity & \multicolumn{5}{|c|}{$45 \%(95 \% \mathrm{Cl} 38 \%-52 \%)$} \\
\hline Positive Predictive Value & \multicolumn{5}{|c|}{$6.5 \%(95 \% \mathrm{Cl} 3.0 \%-12 \%)$} \\
\hline Negative Predictive Value & \multicolumn{5}{|c|}{$99 \%(95 \%$ Cl $95 \%-100 \%)$} \\
\hline
\end{tabular}

three major contributors to the gap in health between Aboriginal and Torres Strait Islander and non-Indigenous Australians. The results demonstrate that clinicians cannot rely on a patient's recall when they report having had been screened within the recommended time interval, since $72 \%, 70 \%$ and $55 \%$ of individuals who had not been screened for diabetes, cholesterol and cervical cancer respectively, according to pathology records, reported having had appropriate screening. These figures indicate a considerable potential for missed opportunity for health gain if health professionals rely on a positive self-report. In addition to individual patient care, there are public health implications if 
disease prevalence is under-estimated or the effectiveness of screening programs is overestimated.

\section{Conversely, for patients who reported that} they had not been screened, the results (NPV was $90 \%$ for diabetes, $70 \%$ for cholesterol and 99\% for cervical cancer) suggested a level of reliability of clinical utility: few resources would be wasted by relying on a negative self-report, and to test all patients who report that they have not been screened. For the future, there are practical implications from the finding that patients who have been tested appropriately for diabetes are highly likely to have correct recall (94\%). This is also true for a Pap test (90\%) although the numbers are much smaller. For cholesterol testing, accurate recall was still reasonably high at $83 \%$.

Best endeavours were undertaken to minimise the effect of confounding variables in this study. There was potential for patients to receive primary health care from practices other than the ACCHS, where the study was based, and so could have been screened elsewhere. This was reduced by ensuring patients were active clients for the period in which routine screening should have occurred. The study also assumed there were no data errors in the patient information management system (Communicare), nor multiple records for the same patient. Communicare is used by many ACCHSs across Australia and is considered to be reliable enough for reporting on clinical performance to the Australian Government as part of the national key performance indicators for Indigenous-specific primary health care services programs.

These data and the conclusions of this study are confined to the study population: Aboriginal and Torres Strait Islander Australians attending a metropolitan ACCHS. However, it is likely that the findings could be generalised to Aboriginal and Torres Strait Islander populations attending other ACCHSs across Australia, particularly in metropolitan areas. The value of a negative self-report and the accuracy of recall in those appropriately screened are also likely to be relevant to non-Indigenous patients and practices. Importantly, the data indicate the unreliability of a positive self-report: $60 \%$ of patients who reported being screened for diabetes, $53 \%$ who reported being screened for cholesterol and $94 \%$ who reported being screened for cervical cancer within recommended guidelines were inadequately screened.
This study was limited to a relatively small sample size. The small numbers of women with a recorded Pap test, and wide confidence intervals, indicate these data should be interpreted with caution. The findings do support the need for similar replication studies given the reliance on selfreported data in policy and practice decisionmaking in primary health care in Australia.

In conclusion, self-reported information is commonly relied on to deliver important preventive health care activities such as screening for chronic conditions. Given the high prevalence of diabetes, cardiovascular disease and cancer among Aboriginal and Torres Strait Islander people, regular screening according to evidence-based guidelines is critical for optimising opportunities to prevent disease and intervene early when disease is detected. In Australia, there are few central registries to provide objective data regarding the screening history of individual patients, and existing registries experience low rates of Indigenous identification which limits the ability for population level analysis on screening rates. ${ }^{29}$

The study findings highlight the importance of finding strategies to increase the accuracy of patient self-report regarding screening history, such as shared electronic health records, improved communication with patients and health literacy.

\section{References}

1. Vos T, Barker B, Begg S, Stanley L, Lopez AD. Burden of disease and injury in Aboriginal and Torres Strait Islander Peoples: The Indigenous health gap. Int $J$ Epidemiol. 2008;38(2):470-7.

2. National Aboriginal Community Controlled Health Organisation. National Guide to a Preventive Health Assessment for Aboriginal and Torres Strait Islander People. 2nd ed. South Melbourne (AUST): Royal Australian College of General Practitioners; 2012.

3. Bowman JA, Redman S, Dickinson JA, Gibberd R Sanson-Fisher RW.The accuracy of Pap smear utilization self-report (a methodological consideration in cervical screening research). Health Serv Res. 1991;26:97-107.

4. Degnan D, Harris R, Ranney J, Quade D, Earp JA, Gonazlez $J$. Measuring the use of mammography (two methods compared). Am J Public Health. 1992:82:1386-8.

5. Zapka JG, Bigelow C, Hurley T, Ford LD, Egelhofer J, Cloud WM, et al. Mammography use among sociodemographically diverse women (the accuracy of self-report). Am J Public Health. 1996;86:1016-21.

6. King ES, Rimer BK, Trock B, Balshem A, Engstrom P. How valid are mammography self-reports? Am JPublic Health. 1990;80:1386-8.

7. Fulton-Kehoe D, Burg MA, Lane DS. Are self-reported dates of mammograms accurate? Public Health Rev. 1992;20:233-40.

8. Sawyer JA, Earp JA, Fletcher RH, Daye FF, Wynn TM. Accuracy of women's self-report of their last Pap smear. Am J Public Health. 1989;79:1036-7.

9. Cronin KA, Miglioretti DL, Krapcho M, Yu B, Geller BM, Carney PA, et al. Bias associated with self-report of prior screening mammography. CancerEpidemiolBiomarkers Prev. 2009;18(6):1699-705.
10. Rauscher GH, Johnson TP, Cho Yl, Walk JA. Accuracy of self-reported cancer-screening histories: A meta-analysis. Cancer Epidemiol Biomarkers Prev. 2008;17(4):748-57.

11. Martin L, Leff M, Calonge N, Garrett C, Nelson DE. Validation of self-reported chronic conditions and health services in a managed care population. $\mathrm{Am} J$ Prev Med. 2000;18(3):215-8

12. Barratt $A$, Cockburn J, Smith D, Redman S. Reliability and validity of women's recall of mammographic screening Aust NZJ Public Health. 2000;24:79-81.

13. Bowman JA, Redman S, Dickinson JA, Gibberd R, Sanson-Fisher RW.The accuracy of Pap smear utilization self-report: A methodological consideration in cervical screening research. Health Serv Res. 1991;26:97-107.

14. Bowman JA, Sanson-Fisher R, Redman S. The accuracy of self-reported Pap smear utilisation. Soc Sci Med. 1997;44:969-76.

15. Newell S, Girgis A, Sanson-Fisher R, Ireland M. Accuracy of patients' recall of Pap and cholesterol screening. Am J Public Health. 2000;90(9):1431-5.

16. Suarez L, Goldman DA, Weiss NS. Validity of Pap smear and mammogram self-reports in a low-income Hispanic population. Am J Prev Med. 1995;11:94-8.

17. McKenna MT, Speers M, Mallin K, Warnecke R. Agreement between patient self-reports and medical records for Pap smear histories. Am J Prev Med. 1992:8:287-91.

18. Johnson CS, Archer J, Campos-Outcalt D. Accuracy of Pap smear and mammogram self-reports in a southwestern native American tribe. Am J Prev Med. 1995; 11:360-3.

19. Liao $Y$, Tucker $P$, Okoro CA, Giles WH, Mokdad AH, Harris VB. REACH 2010 surveillance for health status in minority communities - United States, 2001-2002. MMWR Surveill Summ. 2004;53(6):1-36.

20. Gilliland FD, Mahler R, Hunt WC, Davis SM. Preventive health care among rural American Indians in New Mexico. Prev Med. 1999;28(2):194-202.

21. Gilligan C, Sanson-Fisher R, Eades S, Wenitong M, Panaretto K, D'Este C. Assessing the accuracy of selfreported smoking status and impact of passive smoke exposure among pregnant Aboriginal and Torres Strait Islander women using cotinine biochemical validation. Drug Alcohol Rev. 2010;29(1):35-40.

22. Gwynn JD, Hardy LL, Wiggers JH, Smith WT, D'Este CA Turner N. The validation of a self-report measure and physical activity of Australian Aboriginal and Torres Strait Islander and non-Indigenous rural children. Aust NZJ J Public Health. 2010;34 Suppl 1:57-65.

23. Stewart JM, Sanson-Fisher RW, Eades SJ, D'Este C, Fitzgerald $\mathrm{M}$. The risk status, screening history and health concerns of Aboriginal and Torres Strait Islander people attending an Aboriginal Community Controlled Health Service. Drug Alcohol Rev. 2012:31(5):617-24.

24. Vos T, Barker B, Begg S, Stanley L, Lopez AD. Burden of disease and injury in Aboriginal and Torres Strait Islander Peoples: The Indigenous health gap. Int $J$ Epidemiol. 2008;38(2):470-7.

25. Australian Bureau of Statistics. 4715.0. - National Aboriginal and Torres Strait Islander Health Survey. Canberra (AUST): ABS; 2006.

26. National Aboriginal Community Controlled Health Organisation National Guide to a Preventive Health Assessment in Aboriginal and Torres Strait Islander Peoples. South Melbourne (AUST): Royal Australian College of General Practitioners; 2005.

27. The Royal Australian College of General Practitioners. Guidelines for Preventive Activities in General Practice (The Red Book). 6th ed. South Melbourne (AUST): Royal Australian College of General Practitioners; 2005.

28. United States of America Preventive Services TaskForce. Pocket Guide to Clinical Preventive Services. Washington (DC): USA Department of Health and Human Services; 2006.

29. Cunningham J, Rumbold A, Zhang X, Condon J. Incidence, aetiology, and outcomes of cancer in Indigenous peoples in Australia. Lancet Oncol. 2008:9(6)585-95. 